\title{
Itaconic acid mediates crosstalk between macrophage metabolism and peritoneal tumors
}

\author{
Jonathan M. Weiss, ${ }^{1}$ Luke C. Davies, ${ }^{2}$ Megan Karwan, ${ }^{3}$ Lilia lleva, ${ }^{3}$ Michelle K. Ozaki, ${ }^{4}$ Robert Y.S. Cheng, ${ }^{1}$ \\ Lisa A. Ridnour, ${ }^{1}$ Christina M. Annunziata, ${ }^{4}$ David A. Wink, ${ }^{1}$ and Daniel W. McVicar ${ }^{1}$ \\ 'Cancer and Inflammation Program, Center for Cancer Research, National Cancer Institute (NCI) at Frederick, Frederick, Maryland, USA. ${ }^{2}$ Cardiff University, Division of Infection and Immunity, Cardiff, \\ United Kingdom. ${ }^{3}$ Frederick National Laboratory for Cancer Research, Leidos Biomedical Research Inc., Frederick, Maryland, USA. ${ }^{4}$ Women's Malignancies Branch, Center for Cancer Research (CCR), NCI, \\ Bethesda, Maryland, USA.
}

\begin{abstract}
Control of cellular metabolism is critical for efficient cell function, although little is known about the interplay between cell subset-specific metabolites in situ, especially in the tumor setting. Here, we determined how a macrophage-specific (M $\phi$ specific) metabolite, itaconic acid, can regulate tumor progression in the peritoneum. We show that peritoneal tumors (B16 melanoma or ID8 ovarian carcinoma) elicited a fatty acid oxidation-mediated increase in oxidative phosphorylation (OXPHOS) and glycolysis in peritoneal tissue-resident macrophages (pRes $\mathrm{M} \phi)$. Unbiased metabolomics identified itaconic acid, the product of immune-responsive gene 1-mediated (Irg1-mediated) catabolism of mitochondrial cis-aconitate, among the most highly upregulated metabolites in pResM $\phi$ of tumor-bearing mice. Administration of lentivirally encoded Irg1 shRNA significantly reduced peritoneal tumors. This resulted in reductions in OXPHOS and OXPHOS-driven production of ROS in pResM $\phi$ and ROS-mediated MAPK activation in tumor cells. Our findings demonstrate that tumors profoundly alter $\mathrm{pResM} \phi$ metabolism, leading to the production of itaconic acid, which potentiates tumor growth. Monocytes isolated from ovarian carcinoma patients' ascites fluid expressed significantly elevated levels of IRG1. Therefore, IRG1 in pResM $\phi$ represents a potential therapeutic target for peritoneal tumors.
\end{abstract}

\section{Introduction}

The peritoneal cavity is a frequent site of tumor development and metastasis. Ovarian epithelial cancer often spreads to the peritoneal cavity and is treated similarly to primary peritoneal cancer (1). The peritoneal cavity is also home to a heterogeneous population of peritoneal tissue-resident macrophages $(\mathrm{pRes} \mathrm{M} \phi)$ that arise from hematopoietic progenitors and play important roles in homeostasis and immune surveillance $(2,3)$. There is clearly the potential for extensive crosstalk between tumor cells and $\operatorname{pResM} \phi$ within the peritoneal cavity. We hypothesized that peritoneal tumors might profoundly influence the metabolism, and therefore the function, of pResM $\phi$ and specifically sought to characterize those metabolic alterations that in turn regulate tumor progression.

Although $\mathrm{M} \phi$ can mediate important antitumor responses, there is considerable evidence for their role in promoting the initiation, growth, and metastatic spread of many tumors. The ability of $\mathrm{M} \phi$ to produce immunosuppressive cytokines and angiogenic factors as well as promote tumor growth has been well described (4). In the peritoneum, $\mathrm{M} \phi$ depletion can reduce tumor progression, metastasis (5), and ascites fluid formation (6). M $\phi$-targeted

Conflict of interest: The authors have declared that no conflict of interest exists. License: This work is licensed under the Creative Commons Attribution 4.0 International License. To view a copy of this license, visit http://creativecommons.org/ licenses/by/4.0/.

Submitted: December 11, 2017; Accepted: June 12, 2018.

Reference information: / Clin Invest. 2018;128(9):3794-3805.

https://doi.org/10.1172/JCI99169. therapies are thus promising strategies for the treatment of ovarian carcinoma and peritoneal tumors.

Although tumor metabolism has been well studied, it is important to define the effects of host-tumor interactions on immune cell metabolism when metabolism-based therapies are used. Tumors have long been known to have high glycolytic rates, as they consume large amounts of glucose and release metabolic products of glycolysis. Glycolytic tumors have been proposed to metabolically restrict $\mathrm{T}$ cells, in part by outcompeting them for nutrients such as glucose (7). Moreover, tumor-derived metabolites, such as lactic acid, have been implicated in polarizing $\mathrm{M} \phi$ toward a tumor-promoting phenotype, characterized by high levels of VEGF and arginase (8). M $\phi$ polarization is closely linked to metabolic programming, with alterations in key signaling pathways underlying distinct utilization modes of glucose metabolism (9-14). Classically activated $\mathrm{M} \phi$ exhibit increases in glycolysis mediated through the AKT/mTOR/HIF1 $\alpha$ pathway to regulate the inflammatory phenotype. In contrast, alternatively activated $\mathrm{M} \phi$ have a vastly different metabolic and inflammatory profile promoted by increased oxidative phosphorylation (OXPHOS). Given that cellular metabolism is critical for the regulation of the $\mathrm{M} \phi$ phenotype, tumor-mediated alterations in the expression of $\mathrm{M} \phi$ metabolites clearly have the potential to underlie important differences in the effector function of pResM $\phi$.

Itaconate is among the most selectively and highly upregulated metabolites in classically activated $\mathrm{M} \phi(13,15,16)$. Itaconate mediates antimicrobial functions in $\mathrm{M} \phi$ by inhibiting isocitrate lyase, an enzyme of the glyoxylate shunt used as a bacterial survival mechanism (17). Recently, mitochondria-associated itaconate has 
A
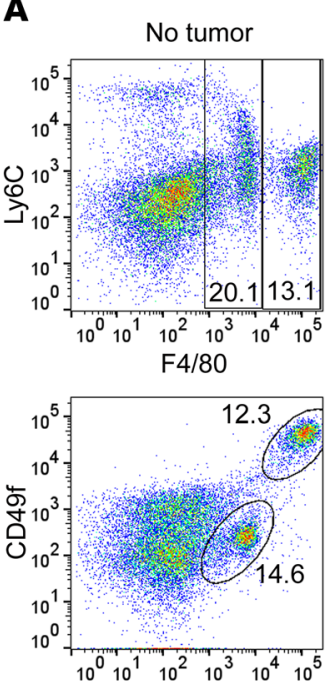

$\mathrm{F} 4 / 80$
B

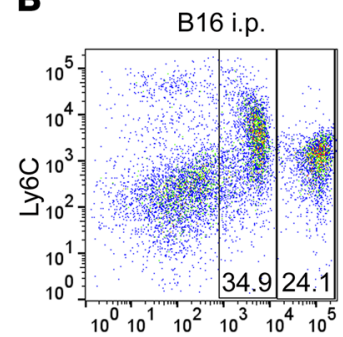

$\mathrm{F} 4 / 80$

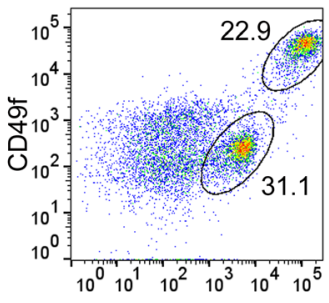

$\mathrm{F} 4 / 80$
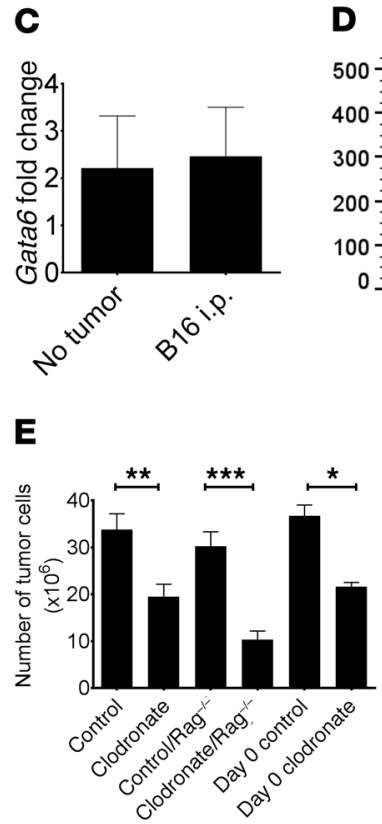
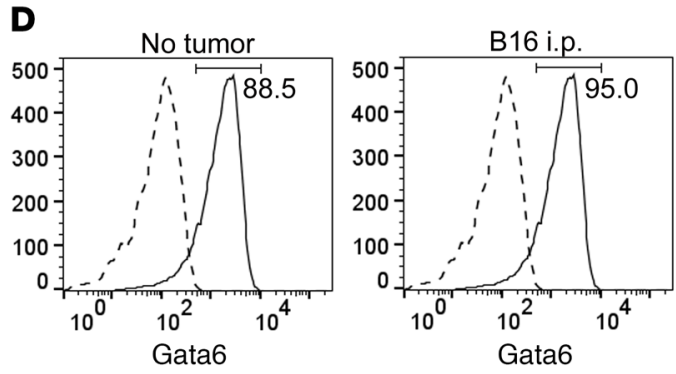

$\mathbf{F}$

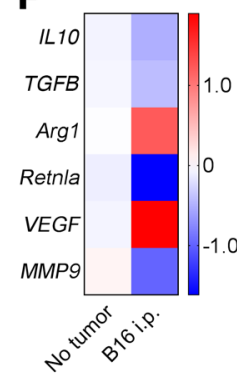

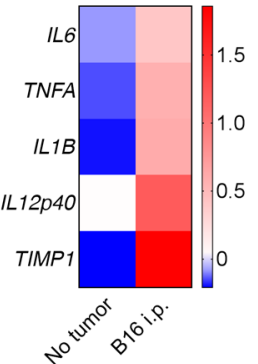

Figure 1. Characterization of peritoneal leukocytes from tumor-bearing hosts. Flow cytometric analysis was performed on the peritoneal lavage cells from (A) naive and (B) B16 tumor-bearing mice. Among F4/80-sorted leukocytes, Gata6 expression was evaluated by (C) qPCR and (D) intracellular flow cytometry. Flow cytometric plots are representative of 6 samples (2 experiments each consisting of triplicate samples). The dotted lines represent isotype control staining. (E) B16 tumor burden was quantified in WT or Rag ${ }^{-/}$mice that received control or clodronate liposomes on day 2 (after tumor) or day 0 (before tumor) ( $n \geq 5) .{ }^{*} P<0.05,{ }^{*} P<0.01$, and ${ }^{* * *} P<0.001$, by ANOVA with Tukey's multiple comparisons test. (F) F4/80-sorted M $\phi$ were evaluated by qPCR for M2 (left) and M1 (right) prototypic gene expression. Triplicate samples were evaluated, with one of the no-tumor samples set to 1.0 as a reference point (heatmaps depict $\log _{2}$-transformed, relative-based results of gene expression; all genes shown were significantly altered in the B16 group to at least $P<0.05$ as compared with the no-tumor group; unpaired Student's $t$ test). Data represent the mean \pm SEM.

emerged as an important metabolic regulator of $\mathrm{M} \phi$ function by regulating the glycolytic pathway and promoting succinate accumulation during mitochondrial respiration $(18,19)$. Mammalian production of itaconate, or itaconic acid, is catalyzed by immuneresponsive gene 1 ( Irg1), which is transcriptionally regulated by IFN- $\gamma$, TNF- $\alpha$, and LPS stimulation $(20,21)$. IRG1 catabolizes fatty acid-derived metabolites, which are necessary energy substrates for OXPHOS in $\mathrm{M} \phi$ and OXPHOS-associated ROS production by mitochondria $(14,22-24)$. The IRG1 product itaconate thus links metabolism with proinflammatory processes and ROS production in $\mathrm{M} \phi$ through alterations in fatty acid oxidation and OXPHOS.

ROS encompass a wide range of molecules such as hydrogen peroxide, superoxide, hydroxyl radicals, and peroxynitrite. In addition to serving as a critical component of $\mathrm{M} \phi$ bactericidal activity, ROS production can have profound effects on inflammatory responses and tumor progression. ROS activate numerous transcription factors (e.g., NF-кB, HIF1 $\alpha$, STAT3, and AP-1), resulting in a complex association between ROS expression and tumor progression (25-27). Although ROS are a critical component of many antitumor responses, ROS expression has also been associated with the promotion of tumor cell growth and survival, in part through enhanced expression and activation of $\mathrm{NF}-\mathrm{\kappa B}$ and MAPK $(26,28,29)$.

In this study, we demonstrate that itaconate is among the most highly upregulated metabolites in $\mathrm{pResM} \phi$ from tumor-bearing mice and that an increase in its levels plays an important role in tumor progression. Targeted blockade of tumor-induced itaconate in pResM $\phi$ using a lentivirus-based shRNA approach demon- strates that itaconate promotes tumor growth via OXPHOS-driven ROS expression in resident $\mathrm{M} \phi$ and concomitant ROS-mediated MAPK activation in tumor cells. Our data suggest that metabolic targeting of IRG1 and itaconate in $\mathrm{M} \phi$ may be effective for the control of ROS levels and ROS-driven tumor progression. An advantage of our approach is the feasibility of specifically targeting resident $\mathrm{M} \phi$-associated itaconate and ROS for efficient control of peritoneal tumors.

\section{Results}

Resident $M \phi$ mediate protumor effects in the peritoneum. Hypothesizing that progressing tumors extensively interact with $\mathrm{pRes} \phi \phi$, we first compared macrophages $(\mathrm{M} \phi)$ from naive and tumorbearing mice. $\mathrm{F} 4 / 80^{+} \mathrm{M} \phi$ from peritoneal lavages could be separated into 2 populations on the basis of their relative levels of $\mathrm{F} 4$ / 80 and CD49f expression (Figure 1A). The $2 \mathrm{M} \phi$ populations are consistent with larger (LPM) and smaller (SPM) pResM $\phi$ populations that have been described previously $(2,30)$. The relative proportion of LPMs and SPMs was similar between control mice and mice bearing peritoneal tumors, although the overall frequency of $\mathrm{F} 4 / 80^{+} \mathrm{M} \phi$ was increased in tumor-bearing mice (Figure $1 \mathrm{~B}$ and Supplemental Figures 1, A-D; supplemental material available online with this article; https://doi.org/10.1172/JCI99169DS1), with the total number of leukocytes remaining similar between control and tumor-bearing mice. To further characterize $\mathrm{pResM} \phi$, we evaluated their expression of Gata-binding factor 6 (Gata6), a transcription factor shown to be critical for their phenotype (31, 32). Gata6 mRNA (Figure 1C) and protein expression levels were 
A
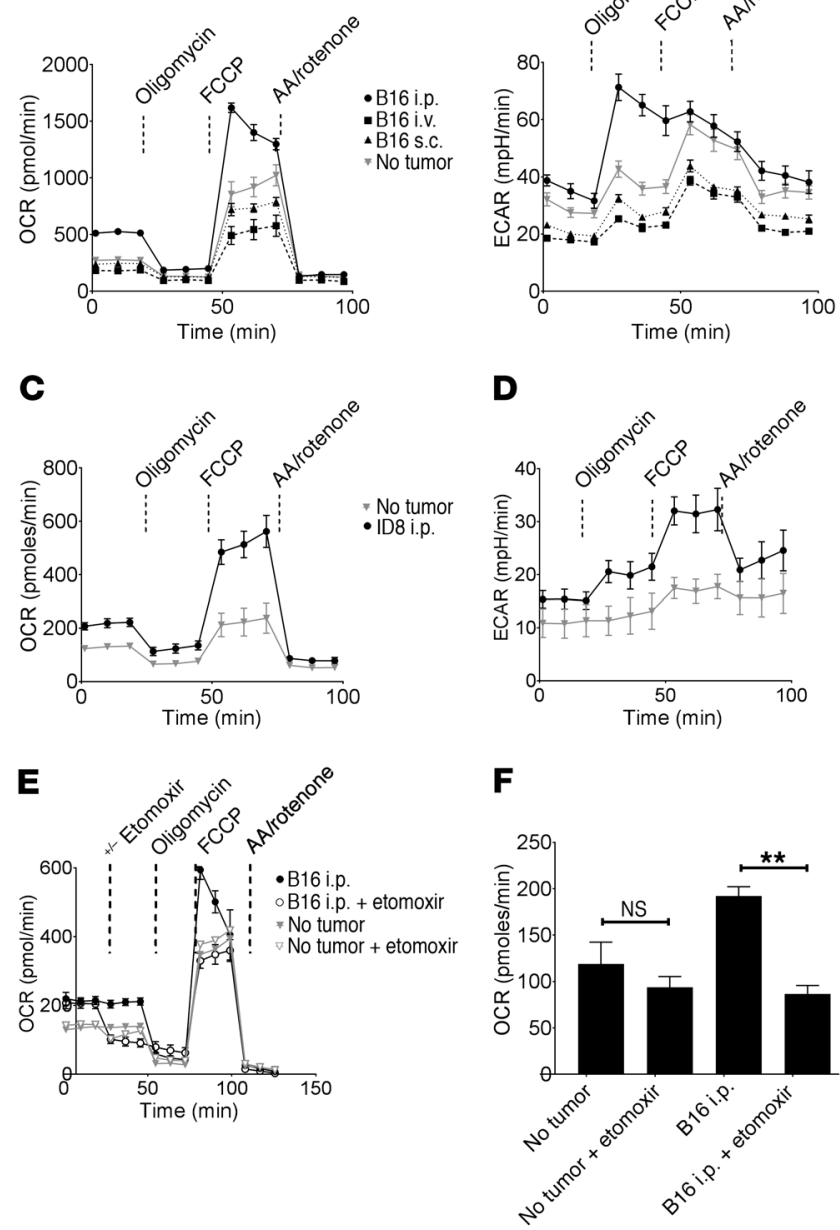

Figure 2. Tumors increase oxidative and glycolytic metabolism in pResM $\phi$. Extracellular flux analysis of F4/80-sorted pResM $\phi$ from nontumor-bearing control mice or mice inoculated with $\mathrm{B} 16$ via the indicated routes were analyzed. The (A) OCR and (B) ECAR were graphed over time as indicators of OXPHOS and glycolysis, respectively. Drugs were injected into the ports at the indicated time points. F4/80-sorted pResM $\phi$ from no-tumor-bearing control mice or mice bearing ID8 ovarian carcinoma (day 47) were similarly evaluated for (C) cellular OCR and (D) ECAR. (E) OXPHOS of peritoneal $\mathrm{M} \phi$ from control and $\mathrm{B} 16$ tumor-bearing mice were evaluated following injection of etomoxir into the first port at the indicated time point. (F) Basal OCRs for the treatment groups were graphed. ${ }^{* *} P<0.01$, by ANOVA with Tukey's multiple comparisons test. All plots are representative of 3 experiments. Data represent the mean \pm SEM. AA, Antimycin A.

similar between $\mathrm{pRes} \mathrm{M} \phi$ from control and tumor-bearing mice (Figure 1D and Supplemental Figure 1E).

Numerous studies have ascribed a protumor phenotype for tumor-associated $\mathrm{M} \phi$ (4). Consistently, we found that specific depletion of peritoneal $\mathrm{M} \phi$ ( $>90 \%$ depletion) 2 days after tumor inoculation resulted in a significantly reduced tumor burden (Figure 1E). We obtained similar results using Rag1-/- mice, indicating that the protumor effects were independent of $\mathrm{T}$ and $\mathrm{B}$ lymphocytes. Moreover, the reductions in tumor burden were indistinguishable when the clodronate-depleting agent was administered prior to tumor inoculation (day 0), suggesting that the pResM $\phi$ populations present in naive mice already have tumor-promoting potential. The protumor phenotype of resident $\mathrm{M} \phi$ could not be attributed to a shift between pro- and antiinflammatory phenotypes. Although arginase 1 (Arg1) and Vegf increased, the expression of most prototypically protumor genes we evaluated was decreased in cells from tumor-bearing mice (Figure $1 \mathrm{~F}$, left), with a concomitant increase in all proinflammatory genes evaluated (Figure 1F, right). These data indicate that $\mathrm{pRes} M \phi$ exhibit a tumor-promoting phenotype that cannot be ascribed to a generalized skewing in the expression of prototypic immunosuppressive and tumor-promoting molecules. Indeed, it has been suggested that classifying $\mathrm{M} \phi$ as polarized and ascribing $\mathrm{M} \phi$ function on the basis of pro- and antiinflammatory gene expression is an oversimplification and that consideration of gene function is more meaningful (33).

The metabolism of resident $M \phi$ from tumor-bearing mice is increased as a result of fatty acid oxidation. We hypothesized that metabolic alterations in $\mathrm{pResM} \phi$ may underlie their protumor phenotype. We therefore evaluated the extracellular flux of pResM $\phi$ isolated from control and tumor-bearing mice. $\mathrm{pRes} \mathrm{M} \phi$ from mice bearing peritoneal B16 tumors (i.p. inoculation) had dramatically elevated basal and maximal oxygen consumption rates (OCRs) (Figure 2A) and extracellular acidification rates (ECARs) (Figure 2B) as compared with those of control mice or mice inoculated with B16 tumors via distant routes (i.v. or s.c.). This suggests that increased $\mathrm{pResM} \phi$ metabolism is locally modulated by peritoneal tumors. We observed similar results using the ID8 ovarian carcinoma cell line (Figure 2, C and D). Cells can use diverse mechanisms to increase OXPHOS, however, it has been reported that antiinflammatory M $\phi$ use fatty acids to support OXPHOS (34). We therefore hypothesized that $\mathrm{pResM} \phi$ might use fatty acids to support their protumor phenotype and confirmed that fatty acid oxidation was an important component of tumor-mediated increases in OXPHOS, as treatment with etomoxir reduced basal OCRs to levels observed in non-tumor-bearing mice (Figure 2, E and F).

Itaconic acid is the most highly upregulated metabolite in $p R e s M \phi$ from tumor-bearing mice. Since $\mathrm{M} \phi$ metabolism was significantly increased by tumors, we next investigated whether altered metabolite levels in $\mathrm{pResM} \phi$ are associated with tumor promotion. Using untargeted profiling of primary metabolites in purified $\mathrm{pResM} \phi$, we identified several metabolites that were significantly upregulated in B16 and ID8 tumor-bearing mice as compared with naive mice (Figure 3, A and B, respectively). Among the most highly upregulated metabolites were lactic acid, previously implicated in $\mathrm{M} \phi$ polarization (8), ornithine and polyamines such as putrescine, implicated in arginine metabolism and tumor promotion (35), and, somewhat surprisingly, itaconic acid, which was the most highly upregulated metabolite ( $>15$-fold). Itaconate accumulation is catalyzed by the enzyme encoded by $\operatorname{Irg} 1$ (20); accordingly, we found that Irg1 mRNA expression was significantly upregulated in $\mathrm{pResM} \phi$ from mice bearing B16, 3LL, or MC38 tumors (Figure 3C) as well as in mice bearing ID8, IG10, or IF5 ovarian carcinomas (Figure 3D). Ingenuity Pathway Analysis (IPA) (QIAGEN; https://www. qiagenbioinformatics.com/products/ingenuitypathway-analysis) of our metabolomics data confirmed not only that itaconate was the most highly upregulated metabolite but that several saturated fatty acids were also significantly downregulated (Table 1), consistent with the association between itaconic acid production and 


\section{Table 1. IPA of the metabolomics data}

\begin{tabular}{lc} 
Molecules & Expression fold change \\
Itaconic acid & $\uparrow 15.922$ \\
L-asparagine & $\uparrow 11.363$ \\
2-oleoylglycerol & $\uparrow 7.255$ \\
Thymidine & $\uparrow 7.162$ \\
Thymine & $\uparrow 7.156$ \\
Ethanolamine & $\uparrow 6.905$ \\
L-alanylalanine & $\uparrow 6.354$ \\
L-lysine & $\uparrow 6.264$ \\
L-ornithine & $\uparrow 5.996$ \\
(S)-2,4-diaminobutanoic acid & $\downarrow-6.220$ \\
Octanoic acid & $\downarrow-5.930$ \\
Decanoic acid & $\downarrow-4.692$ \\
Dihydroxyacetone & $\downarrow-4.554$ \\
1,5-anhydroglucitol & $\downarrow-4.520$ \\
Lauric acid & $\downarrow-2.649$ \\
1-palmitoylglycerol & $\downarrow-1.786$ \\
Glutaric acid & $\downarrow-1.629$ \\
Benzoic acid & $\downarrow-1.518$ \\
Isomaltose & $\downarrow-1.311$ \\
Unbiased metabolomic analysis was performed on F4/80-sorted pResM $\phi$ \\
from control and B16 melanoma-bearing mice. Each group contained at \\
least 5 replicate samples. The data sets were analyzed using IPA software \\
(QIAGEN) to obtain a list of the top-10 upregulated and downregulated \\
molecules in the tumor-bearing group as compared with the control group. \\
\hline
\end{tabular}

fatty acid degradation (23). Western blotting confirmed IRG1 protein expression in $\mathrm{pResM} \phi$ from tumor-bearing mice, but IRG1 was not detected in B16 or ID8 tumor lysates, further confirming that the IRG1 expression in our system was due to the $\mathrm{pResM} \phi$ (Figure $3 \mathrm{E})$. In vitro, we observed that Irg1 expression was also upregulated in pResM $\phi$ cocultured with B16 or ID8 tumor cells (Figure 3F). Interestingly, the ability of B16 tumor cells to induce Irg1 expression in $\mathrm{pResM} \phi$ was lost when the tumor cells were separated from the $\mathrm{M} \phi$ by a Transwell insert, suggesting that cell-cell contact is important for B16-mediated Irg1 induction. In contrast, ID8 cells were still capable of inducing Irg1 expression in Transwell conditions, indicating a distinct mechanism of Irg1 induction for these tumor cells that involved soluble factor(s) (Figure 3F). These data suggest that tumors elicit an accumulation of itaconic acid as a result of Irg1 expression in pResM $\phi$.

Irg1 silencing in pResM $\phi$ reduces peritoneal tumor burden. To more directly delineate a possible role for itaconic acid in tumor progression, we used a lentiviral shRNA approach that specifically targets Irg1 expression in $\operatorname{pResM} \phi(3,32)$. Injection of Irg1 shRNA i.p. resulted in a significant reduction of Irg1 expression in F4/80-sorted pResM $\phi$ (Figure 4A). Remarkably, Irg1 shRNA treatment significantly reduced B16 tumor burden in the peritoneum, as determined by cell counting (Figure 4B) and MRI imaging of live tumor-bearing mice (Figure 4, C and D). Likewise, Irg1 shRNA significantly reduced ID8 ovarian carcinoma in the peritoneum (Figure 4, E and F). Although Irg1 targeting in pResM $\phi$ had this profound effect, it did not alter the overall number of $\mathrm{F} 4 / 80^{+} \mathrm{M} \phi$ recovered from the peritoneum (Figure $4 \mathrm{G}$ ) or Gata6 expression by pResM $\phi$ (Figure $4, \mathrm{H}-\mathrm{J})$. Moreover, we confirmed that scram- bled (Figure 4K) and $\operatorname{Irg} 1$ (Figure 4L) shRNA vectors transduced pResM $\phi$ similarly (see also Supplemental Figure 2A) and that EGFP encoded by our vectors was not detected in tumor cells or any host cells other than pResM $\phi$. Additionally, we observed no differences in cytokine gene expression in $\mathrm{pResM} \phi$ transduced with Irg1 shRNA as compared with expression levels in the scrambled control (Supplemental Figure 2B). Although multiple metabolites were increased in tumor-bearing mice, knockdown of other metabolic enzymes, namely arginase 1 and lactate dehydrogenases A and B, did not affect tumor burden (Supplemental Figure 3). To complement the lentivirus-based approach, we also found that adoptive transfer of $\operatorname{Irg1}^{-/}$, but not WT, pResM $\phi$ into clodronate-depleted recipient mice resulted in a similarly significant reduction of peritoneal tumor burden (Figure 4M). The adoptively transferred donor cells comprised more than $90 \%$ of the recovered $\mathrm{pResM} \phi$ in recipient mice, and equivalent yields of total pResM $\phi$ were obtained from mice receiving cells from WT and $\mathrm{Irg1}^{-1-}$ mice (Supplemental Figure 4). Taken together, these data demonstrate that tumor-induced Irg1 expression is required for $\mathrm{pRes} \mathrm{M} \phi$-associated tumor promotion.

Irg1 in pResM $\phi$ regulates OXPHOS. Knowing that itaconic acid can regulate OXPHOS and cell metabolism in other systems (13, 18, 19), we next determined whether Irg1 shRNA treatment alters pResM $\phi$ metabolism and whether those changes could be important for the control of tumors. Treatment of tumor-bearing mice with Irg1 shRNA reduced the basal and maximal OCRs of pResM $\phi$ to levels comparable to those in non-tumor-bearing control mice (Figure 5, A and B). In contrast, we found that Irg1 shRNA had no effect on the basal OCR of pResM $\phi$ from control mice.

We reasoned that reduced OXPHOS by pResM $\phi$ in Irg1 shRNAtreated mice could either be due to direct regulation by resulting itaconic acid or an indirect result of the lower tumor burden in these mice. To distinguish between these 2 possibilities, we increased the tumor dose to a level at which Irg1 shRNA treatment was no longer capable of reducing the tumor burden (Figure 5C). At this higher dose, however, we found that Irg1 shRNA was still capable of reducing $\mathrm{M} \phi$ basal and maximal OCRs (Figure 5D). Thus, the reduced OXPHOS following Irg1 shRNA is not simply due to a reduced tumor burden but rather is a direct effect of the lack of Irg1.

Itaconic acid regulates mitochondrial ROS production in $p$ Res $M \phi$. Recent studies have shown ROS production to be a component of epithelial IRG1-mediated regulation of inflammation (24) and the antimicrobial properties of IRG1 in M $\phi$ lineage cells in a zebrafish model (23). Therefore, we sought to determine whether Irg1 could modulate ROS levels in pResM $\phi$ in the context of tumor regulation. ROS levels were evaluated by several different means. First, we used the cell-permeant dye CM-H2DCFDA that detects hydroxyl, peroxyl, and other ROS within cells. Irg1 shRNA treatment significantly reduced the median expression of CM-H2DCFDA in pResM $\phi$ to levels observed in non-tumor-bearing mice (Figure 5E and Supplemental Figure 5A). Recognizing that CMH2DCFDA, as a general oxidative stress indicator, has certain limitations (36), we also measured MitoSOX Red as a marker of superoxide specifically attributable to mitochondrial function. We consistently found that Irg1 shRNA treatment significantly reduced the median expression of MitoSOX Red to control levels (Figure 5F and Supplemental Figure 5B). Furthermore, we 
A

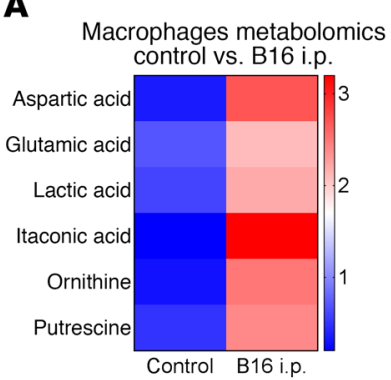

C

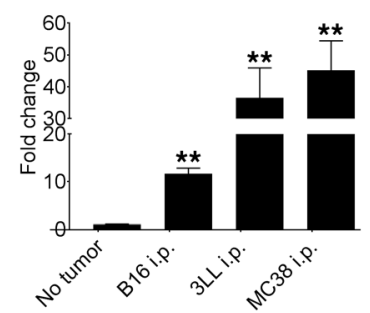

E

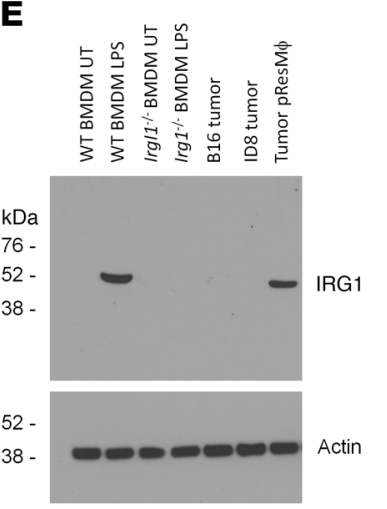

B

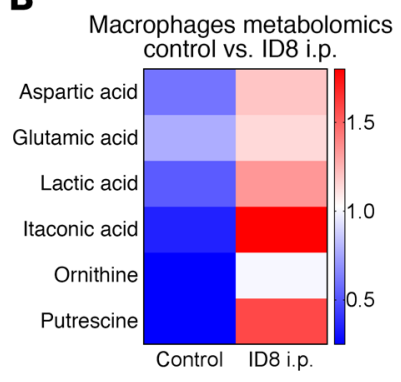

D

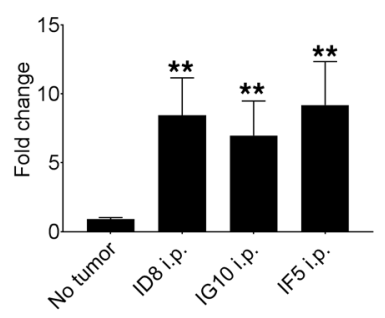

$\mathbf{F}$

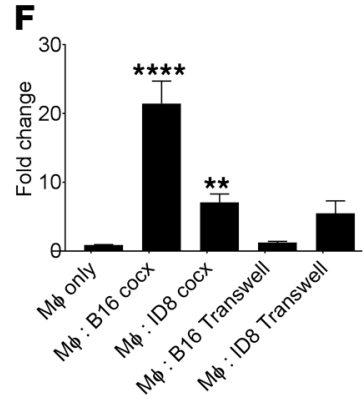

Figure 3. Irg1 and itaconic acid are upregulated in $\mathrm{pRes} M \phi$ by peritoneal tumors. Unbiased metabolomic analysis was performed on F4/80-sorted pResM $\phi$ from control and either (A) B16 melanoma- or (B) ID8 ovarian carcinoma-bearing mice. The mean values from at least 5 replicate samples were $\log _{10}$ transformed and plotted $(P<0.05$ for all metabolites by unpaired Student's $t$ test). Irg1 gene expression was evaluated by QPCR in $\mathrm{F} 4 / 80$-sorted $p$ ResM $\phi$ from control mice and mice bearing either (C) day-9 B16 melanoma, 3LL, or MC38 tumors or (D) day-30 ID8, IG10, or IF5 ovarian carcinomas. Triplicate samples were evaluated, with one of the no-tumor control samples serving as the 1.0 relative reference point. ${ }^{*} P<0.01$, by unpaired Student's $t$ test. (E) IRG1 protein levels in B16 and ID8 tumor lysates and $p$ ResM $\phi$ purified from tumors were determined by Western blotting. Unstimulated or LPS-stimulated bone marrowderived $\mathrm{M} \phi$ from WT and $/ \mathrm{rg}^{1 /-}$ mice were used as controls. (F) pResM $\phi$ were cocultured in vitro with the indicated tumor cells for 48 hours. Irg1 expression was evaluated by qPCR $(n=3)$. cocx, co-culture. ${ }^{*} P<0.01$ and ${ }^{* * *} P<0.0001$, by ANOVA with Tukey's multiple comparisons test. Data represent the mean \pm SEM.

detected increased CM-H2DCFDA levels when pResM $\phi$ from naive WT mice were cocultured in vitro with either B16 or ID8 tumor cells, but not when pResM $\phi$ from $\operatorname{Irg}^{-/-}$mice were used (Figure 5G and Supplemental Figure 5C). Changes in ROS production were further confirmed by analyzing the expression of 11 antioxidant genes whose expression is tied to ROS levels (37). By quantitative PCR (qPCR), we found that the expression of

$\mathrm{Nrf2}$, a key regulatory factor of antioxidant gene expression, as well as that of 7 of 10 other antioxidant genes was significantly downregulated in $\mathrm{pResM} \phi$ from $\mathrm{Irg} 1$ shRNA-treated mice (Figure $5 \mathrm{H})$. The levels of $N r f 2$ expression in pResM $\phi$ from Irg1 shRNAtreated tumor-bearing mice were comparable to those of $\mathrm{pResM} \phi$ from non-tumor-bearing control mice. Extracellular ROS levels were significantly elevated in the peritoneal lavage fluid from tumor-bearing mice as compared with levels in fluid from control mice (Supplemental Figure 6A). Taken together, these data indicate that tumor-induced Irg1 expression promotes mitochondrial ROS levels and subsequent antioxidant activity in pResM $\phi$. Since ROS have been reported to induce cytokine expression and pResM $\phi$ can be important sources of ROS, the lack of changes in their cytokine gene expression in these $\mathrm{pResM} \phi$ in the context of changing ROS levels (Supplemental Figure 2B) was somewhat surprising. Our metabolomic data analysis revealed that $\mathrm{pResM} \phi$ have significantly higher amounts of glutathione as compared with bone marrow-derived $M \phi$, which may partly protect them from such ROS-mediated effects (Supplemental Figure 6B).

Itaconic acid-mediated ROS in pResM $\phi$ regulate MAPK activation in tumor cells. We next sought to mechanistically link tumorinduced, Irg1-dependent M $\phi$ ROS levels with tumor promotion. Although ROS have well-described antitumor effects in some settings, it has also been shown to activate pathways promoting tumor growth $(25,28)$. We found that specific Irg1 knockdown in pResM $\phi$ significantly reduced the levels of phosphorylated ERK (p-ERK) in peritoneal B16 tumors (Figure 6A). We also found that clodronate-mediated removal of pResM $\phi$ reduced $\mathrm{p}$-ERK levels to a similar degree in peritoneal tumors (Figure 6B). Treatment of tumor-bearing mice with the antioxidant $N$-acetylcysteine (NAC) significantly reduced p-ERK levels in tumor lysates (Figure 6C) and the peritoneal tumor burden (Figure 6D). Furthermore, p-ERK was significantly elevated in B16 tumor cells cocultured in vitro with WT, but not $\operatorname{Irg1} 1^{-}$, pResM $\phi$ (Figure 6E), and treatment of B16 and ID8 tumors with PD98059, a selective inhibitor of the MAPK cascade, significantly inhibited tumor cell proliferation (Supplemental Figures 6, $C$ and D). Taken together, these findings suggest that tumorinduced Irg1 and subsequent itaconic acid regulate peritoneal tumors, at least in part, through alterations in M $\phi$-specific ROS.

Expression of Irg1 in monocytes associated with human peritoneal tumors. Having established a paradigm whereby tumorinduced Irg1 in pResM $\phi$ results in fatty acid-mediated increases in ROS that in turn activate tumor cells in mice, we asked whether IRG1 expression might be detected in human ovarian cancers. To that end, we isolated the cellular components from the peritoneal ascites fluid of 8 women with ovarian carcinoma and assayed for the expression of IRG1. In parallel, we assayed these samples for CD14 ${ }^{+}$monocytes by flow cytometry. We observed a significant association between the number of $\mathrm{CD}_{1} 4^{+}$monocytes in each bulk cell sample and IRG1 levels, suggesting that these cells were expressing IRG1 (Figure 7A). Consistent with that notion, we found that $I R G 1$ expression was significantly $(P<0.05)$ elevated in isolated monocytes from all 11 patients as compared with expression levels in non-monocyte cell fractions (Figure 7B). Furthermore, IRG1 expression was significantly elevated in $\mathrm{CD} 14^{+}$monocytes isolated from patients' ascites as compared with blood circulating monocytes from either healthy individuals or patients (Figure 7C). 
A

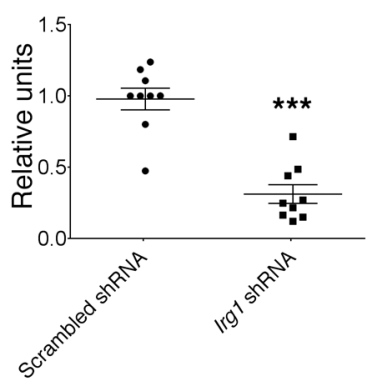

B

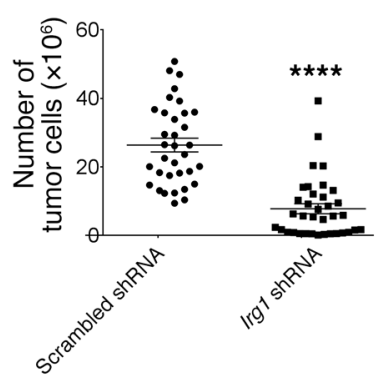

E

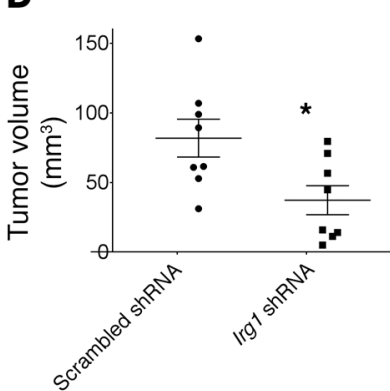

G
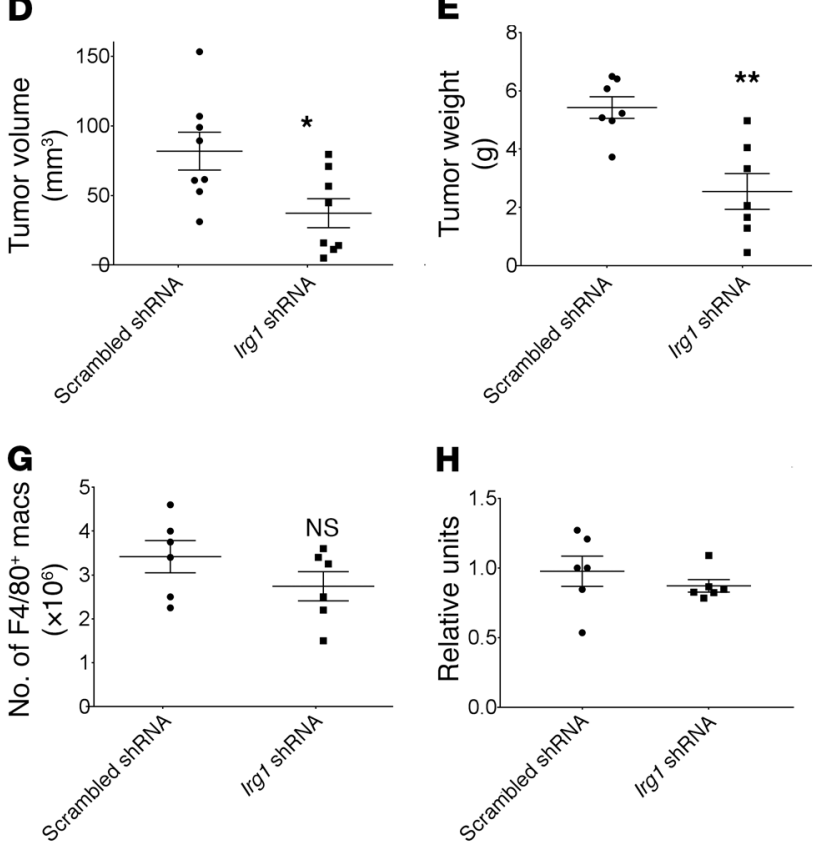

H

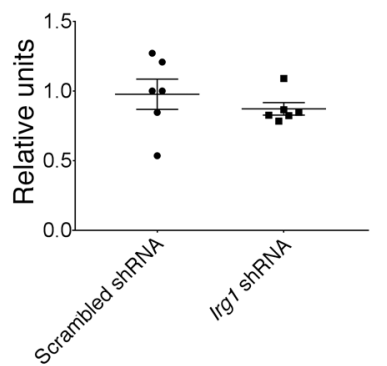

C

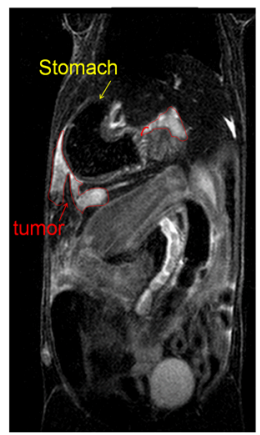

Scrambled shRNA

$\mathbf{F}$

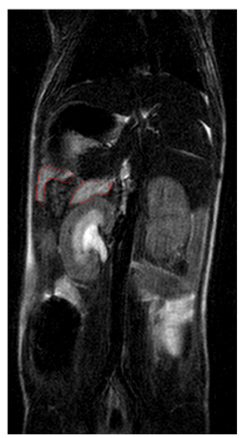

$\operatorname{lrg} 1$ shRNA

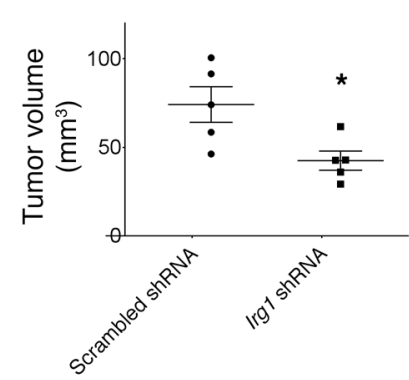

I

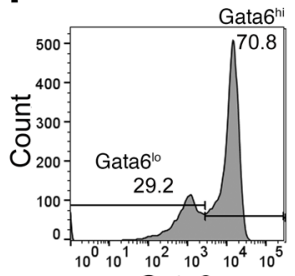

Gata6

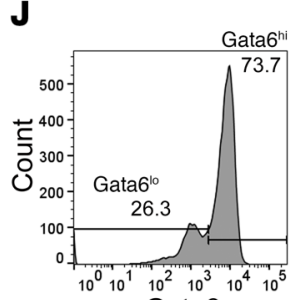

Gata6

M

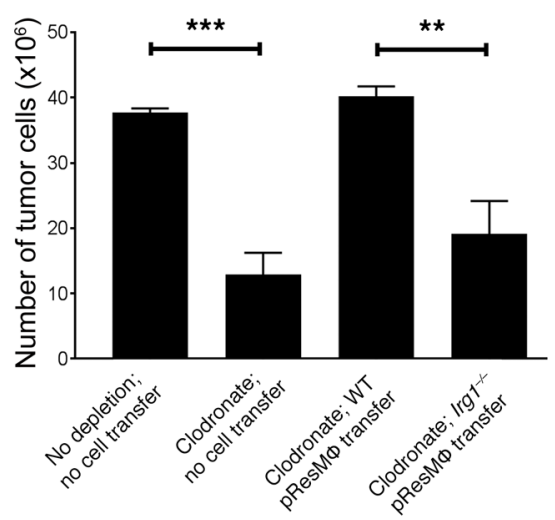

Figure 4. Irg1 silencing in $\mathrm{pRes} \mathrm{M} \phi$ reduces peritoneal tumor burden. B16 tumor-bearing mice were treated with lentiviral shRNA (scrambled control or Irg1 silencing). (A) Gene expression of Irg1 was evaluated by qPCR $(n=9)$. (B) B16 tumor burden was quantitated and (C) evaluated by MRI of live tumor-bearing mice (MRI images are representative of 8 mice). (D) Tumor volumes were calculated by computing the tumor area on each plane and multiplying by the thickness of each slice $(n=8)$. The tumor weight $(\mathbf{E})$ and volume $(\mathbf{F})$ of ID8 ovarian carcinoma-bearing mice were measured $(n \geq 5)$. (G) Total numbers of F4/80+ $p R e s M \phi$ were comparable among 6 scrambled shRNA and Irg1 shRNA recipient mice. Gata6 gene expression by qPCR (H) and protein levels were indistinguishable among scrambled (I) and $\operatorname{lrg} 1$ (J) shRNA recipient mice. A similar uptake of lentiviral shRNA was confirmed by EGFP visualization in F4/80+ $p R e s M \phi$ isolated from mice receiving scrambled (K) or $\operatorname{lrg} 1$ (L) shRNA constructs. All FACS plots are representative of at least 6 mice per group. (M) Clodronate-depleted CD45.1 congenic mice (5 mice/group) were inoculated i.p. with peritoneal lavage cells from WT or $/ \mathrm{rg} 1^{-1-}$ mice 1 day prior to tumor inoculation, and the B16 tumor burden was quantitated. Data represent the mean $\pm \mathrm{SEM} .{ }^{*} P<0.05$, ${ }^{* *} P<0.01$, ${ }^{* *} P<0.001$, and ${ }^{* * * *} P<0.0001$, by Mann-Whitney $U$ test. 
A

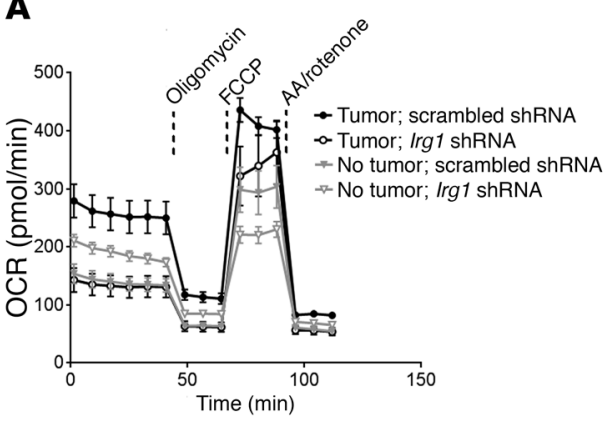

B

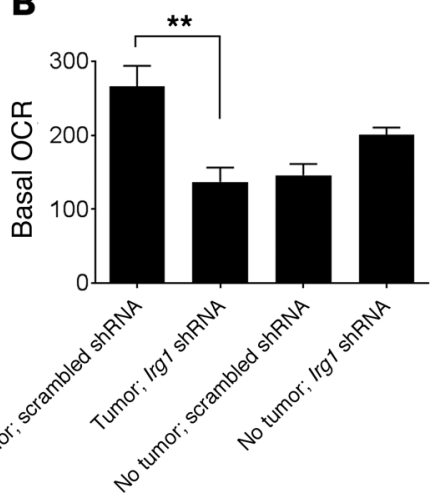

C

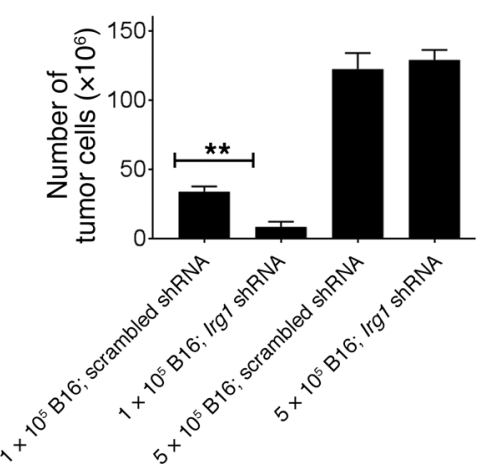

E

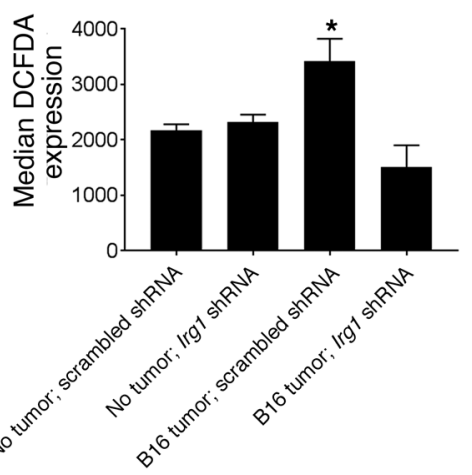

G

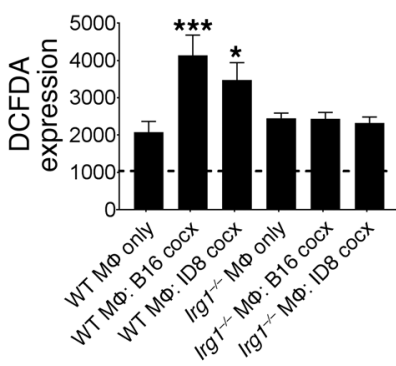

D ${ }^{600}{ }^{2} \quad-1 \times 10^{5} \mathrm{~B} 16$; scrambled shRNA

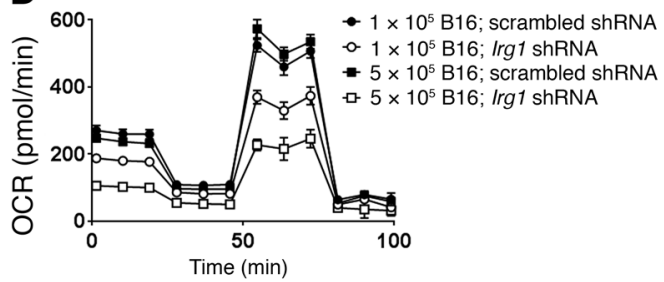

$\mathbf{F}$

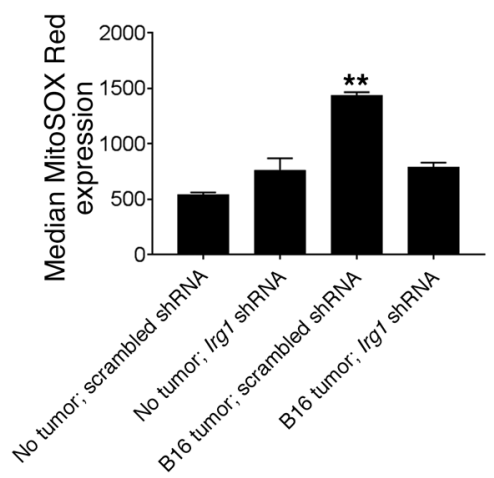

H

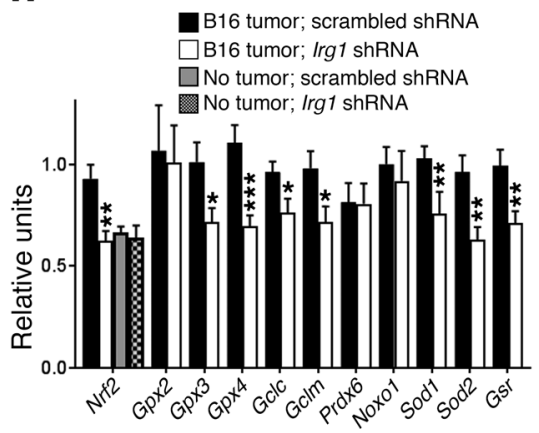

Figure 5. Itaconate regulates OXPHOS and mitochondrial ROS expression in pResM $\phi$. Extracellular flux was analyzed on F4/80-sorted pResM $\phi$ from B16 tumor-bearing mice receiving either scrambled or Irg1 shRNA (A). The OCR over time (A) and the basal OCR (B) were graphed. Data are representative of 3 experiments. ${ }^{* *} P<0.01$, by ANOVA with Tukey's multiple comparisons test. Tumor burden (C) and pResM $\phi$ extracellular flux (D) were evaluated in mice that received $1 \times 10^{5}$ or $5 \times 10^{5}$ B16 tumor cells and either scrambled or Irg1 shRNA $(n=5) .{ }^{* *} P<0.01$, by ANOVA with Tukey's multiple comparisons test. pResM $\phi$ ROS production was assessed by measuring the median CM-H2DCFDA (E) and MitoSOX Red (F) expression by flow cytometry $(n=6) .{ }^{*} P<0.05$ and ${ }^{* *} P<0.01$, by ANOVA with Tukey's multiple comparisons test. (G) pResM $\phi$ from WT or $/ \mathrm{rg}^{1 /-}$ mice were cocultured in vitro with the indicated tumor cells for 48 hours. Median CM-H2DCFDA expression was evaluated by flow cytometry $(n=3)$. The dotted line denotes no DCFDA control staining. ${ }^{*} P<0.05$ and ${ }^{* *} P<0.001$, by ANOVA with Tukey's multiple comparisons test. (H) Gene expression levels modulated by oxidative stress and ROS in mice that received scrambled or Irg1 shRNA were evaluated by qPCR $(n=14)$. ${ }^{*} P<0.05$, ${ }^{* *} P<0.01$, and ${ }^{* *} P<0.001$, by Mann-Whitney $U$ test. Data represent the mean \pm SEM
IRG1 protein was detected in $\mathrm{CD} 14^{+}$monocytes from patients with ovarian carcinoma, but not in normal peripheral blood mononuclear cells (PBMCs) (Figure 7D). These data suggest that myeloid cells associated with human ovarian cancers express IRG1, which positions them to promote tumor growth through itaconate production.

\section{Discussion}

We show for the first time to our knowledge that itaconic acid is a critical metabolic component underlying the crosstalk between tumors and tumor-associated $\mathrm{M} \phi$. Itaconic acid levels were dramatically upregulated in $\mathrm{pResM} \phi$ from tumor-bearing mice, and 
A
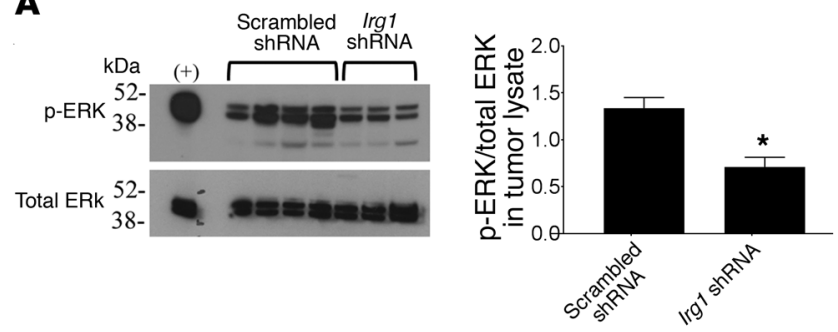

B
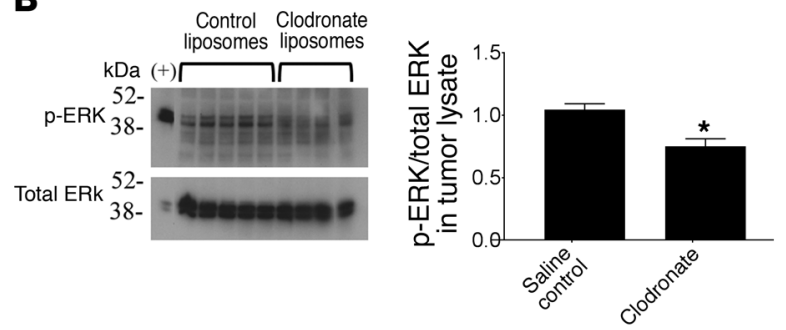

C
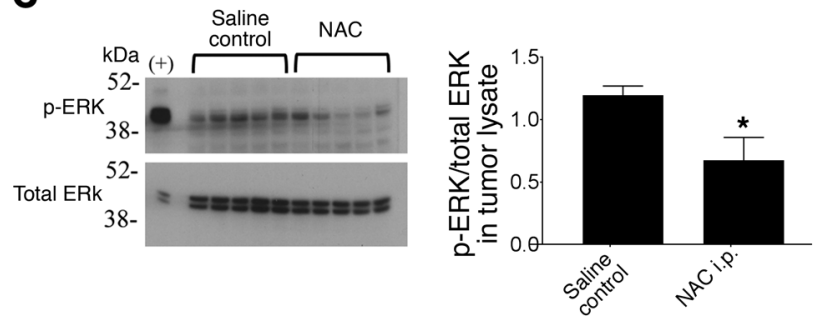

D

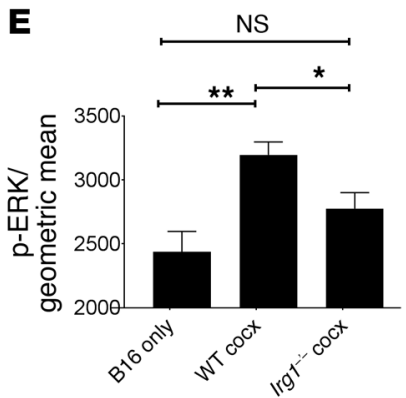

Figure 6. $\mathrm{pResM} \phi$ regulate MAPK activation in peritoneal tumors via itaconate and ROS. B16 tumor lysates were prepared as described in Methods and analyzed by Western blotting for expression of p-ERK and total ERK. (A) Tumor lysates from mice that received scrambled or Irg1 shRNA constructs were compared $(n \geq 4) .{ }^{*} P<0.05$, by Mann-Whitney $U$ test. (B) Tumor lysates from mice that received control or clodronate liposomes were compared. ${ }^{*} P<0.05$, by Mann-Whitney $U$ test. (C) Tumor lysates from saline control- or NAC-treated mice were analyzed $(n=5)$. ${ }^{*} P<0.05$, by Mann-Whitney $U$ test. (D) NAC i.p. treatment reduced the number of B16 tumor cells $(n=10) .{ }^{* *} P<0.01$, by Mann-Whitney $U$ test. (E) B16 tumor cells were cultured in vitro alone or in coculture with the indicated pResM $\phi$. After 48 hours, tumor cells, gated by $\mathrm{CD} 146^{+} \mathrm{F} 4 / 80^{-}$expression, were analyzed by flow cytometry for intracellular $p$-ERK expression. ${ }^{*} P<0.05$ and ${ }^{*} P<0.01$, by ANOVA, corrected for multiple comparisons. Data represent the mean \pm SEM.

this metabolite acts as a critical regulator of $\mathrm{M} \phi$ metabolism, ROS production, and tumor progression, as shown in our study. Irg1 expression was induced in $\mathrm{pResM} \phi$ by 6 cancer cell lines, including 3 different ovarian carcinoma cell lines. Our data expand on the importance of metabolic alterations in the tumor microenvironment and identify itaconate as a potential target for perito- neal cancer treatment. The upregulation of OXPHOS by tumorpromoting itaconate was highly localized, since inoculation of mice with tumors at distant sites did not increase the pResM $\phi$ OCRs or ECARs, and coculture experiments revealed a cellcell contact requirement for promotion of itaconate production. Itaconate production was the only metabolic pathway we tested that was capable of significantly regulating tumor burden. Irg1 expression has been reported in several cell types $(24,38-40)$, but in the peritoneal cavity, it is $\operatorname{Irg} 1$ expression by resident $\mathrm{M} \phi$ that promotes tumor growth. It is possible that blood-borne cells enter the peritoneal cavity and take on the resident phenotype, however, our data indicate that infected $\mathrm{pResM} \phi$ remained over the course of the experiment. Although we could not directly examine the resident $\mathrm{M} \phi$ population of patients with advanced cancer, IRG1 expression in patients' monocytes from tumor ascites suggests that this metabolite could be important in the progression of ovarian carcinoma.

Itaconic acid is emerging as an important regulator of $\mathrm{M} \phi$ metabolism and effector function. The mechanisms by which tumors induce Irg1 and itaconic acid remain unclear and warrant further investigation. $\operatorname{Irg} 1$ induction was still observed when TNF-, IFN- $\gamma-$, IL-6-, or TLR4-KO mice were inoculated with tumors, however, we could not rule out the possibility that tumor-derived cytokines influence pResM $\phi$. One possibility may be the induction of hemoxygenase-1, a stress protein recently shown to induce $\operatorname{Irg1}$, which may be associated with tumors and tumor-associated $\mathrm{M} \phi(41,42)$. Once upregulated, itaconic acid is accompanied by increases in fatty acid-driven OXPHOS. This appears to be in contradiction to the succinate dehydrogenaseinhibitory role ascribed to itaconic acid $(18,19)$. However, OXPHOS is principally driven by NADH recycling, which can be fueled through succinate dehydrogenase-independent mechanisms, such as the mitochondrial citrate and malate shuttles (43), which may operate differently in the presence of tumor. Our findings are consistent with those of Hall et al., who showed that Irg1 depletion impaired fatty acid oxidation in M $\phi$-lineage cells (23). Moreover, we found that upregulated itaconic acid in pResM $\phi$ from tumor-bearing mice was associated with reduced levels of several long-chain fatty acids. Their oxidation may help fuel increases in OXPHOS that comprise a major source of cellular ROS $(23,44)$. Thus, itaconate is clearly important for the regulation of intracellular and extracellular ROS production in pResM $\phi$. ERK activation in tumor cells is probably one of many pathways activated by ROS that could potentially regulate tumor growth in vivo. We never detected Irg1 expression in tumor cells, and if Irg1 expression is relatively $\mathrm{M} \phi$ specific, then targeting $\operatorname{Irg} 1$ levels in the peritoneum may be a means to regulate pResM $\phi$-derived ROS without eliciting some of the side effects associated with systemic ROS inhibition.

Several lines of evidence support the hypothesis that pResM $\phi$, rather than newly migrated inflammatory monocytes, mediate the Irg1-mediated protumor effects. We administered lentiviral constructs 1 week prior to tumor inoculation, thus limiting metabolic programming before any tumor-mediated alterations in leukocyte populations could occur. Importantly, these infected $M \phi$ were labeled with EGFP, which remained with this $\mathrm{Gata}^{+}$cell population throughout our tumor studies. Thus, our alterations of itaconate production were 
A
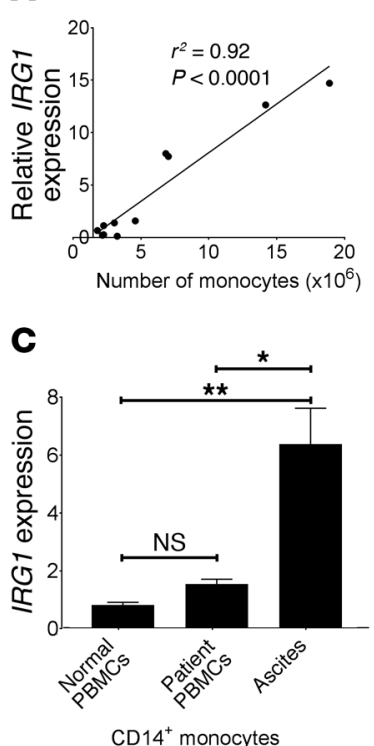

B

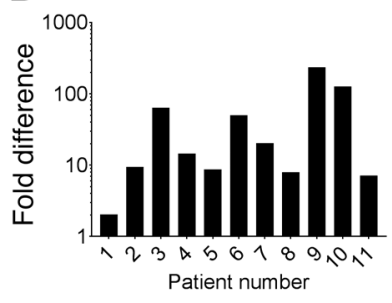

D

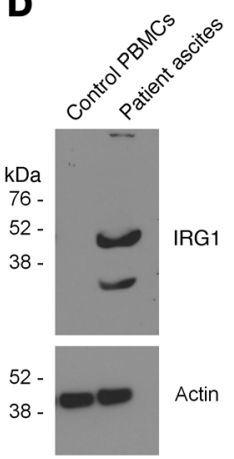

Figure 7. IRG1 is expressed in monocytes associated with human peritoneal tumors. IRC1 expression was evaluated by qPCR using total RNA isolated from cell fractions from 11 human patients with ovarian carcinoma. (A) IRG1 expression levels were established by setting normal PBMC values to 1.0, and the relative levels in ascites monocytes were graphed in relation to the total number of $\mathrm{CD} 45^{+} \mathrm{CD} 14^{+}$monocytes in each sample. Linear regression analysis was performed (GraphPad Prism) to obtain the best curve fit ( $\left.r^{2}=0.93 ; P<0.0001\right)$. (B) The fold difference in IRG1 expression among monocytes and non-monocyte fractions is shown. CD14+ monocytes were purified as described in Methods. For each patient sample, the level of IRG1 expression in the non-monocyte fraction was set to 1.0, and the relative level of IRC1 expression in the corresponding monocyte fraction was graphed $\left(\log _{10}\right)$. Significance was determined by a 1-sample Student's $t$ test using 1.0 as a theoretical mean $(P<0.05)$. (C) IRG1 expression levels in CD14+ PBMCs isolated from 6 healthy volunteers and 5 patients were compared with IRG1 levels in the 11 ascites samples. ${ }^{*} P<0.0$ and ${ }^{* *} P<0.01$, by ANOVA corrected for multiple comparisons. (D) IRG1 protein expression in $\mathrm{CD}_{14}{ }^{+}$monocytes isolated from healthy blood and patients' ascites was analyzed by Western blotting specifically targeted to pResM $\phi$, with results consistent with our previous findings $(3,32)$. Therefore, $\mathrm{pResM} \phi$ specifically facilitate tumor progression in the peritoneum, in large part by tumor-mediated increases in itaconate production. It is becoming increasingly important to understand the regulation of itaconic acid expression in diverse $\mathrm{M} \phi$ subsets. Since myeloidderived suppressor cells (MDSCs), a heterogeneous population of tumor-associated $\mathrm{M} \phi$, utilize fatty acid oxidation to support their ROS production and immunosuppressive phenotype (45), it will be interesting to study metabolic changes in MDSC populations and determine whether targeting itaconic acid might regulate MDSC-associated ROS and MDSC-suppressive potential. Future studies aimed at elucidating the regulation of $\operatorname{Irg} 1$ expression in myeloid cells of different anatomical compartments are warranted.

The identification of itaconic acid as an important regulator of $\mathrm{pResM} \phi$ function raises the intriguing question of how this might be used therapeutically for the control of ovarian and peritoneal tumors. Our results indicate that tumor burden is likely to be an important factor in dictating the success of IRG1-based therapies and that early intervention is important, since $\operatorname{Irg} 1$ intervention was effective at lower, but not higher, tumor burdens. The importance of itaconic acid as an inhibitor of metabolic pathways such as those for isocitrate lyase in bacteria and glycolysis in mammals may yield important clues for its development as a therapeutic target. Irg1-deficient $\mathrm{M} \phi$ have impaired bactericidal capabilities, and certain pathogenic bacteria, such as Yersinia pestis and Pseudomonas aeruginosa, degrade itaconate as a survival mechanism (46). Itaconate metabolism occurs via enzymes that convert itaconate into pyruvate and acetyl-CoA. Mammals have similar mechanisms for degrading itaconate (47) that are probably a means to counteract itaconate-mediated inhibition of glycolysis (48). Our data identify itaconic acid as a potential therapeutic target and support the need for additional research into the multitude of ways itaconate regulates tumor and host cellular responses at differing ratios between $\mathrm{M} \phi$ and tumor burden levels so that the targeting of this metabolite for degradation becomes a potential means by which peritoneal tumors and perhaps other diseases can be treated.

\section{Methods}

Mice. WT C57Bl/6 and CD45.1 (Ptprc ${ }^{\mathrm{a}}$ ) mice were obtained from The Jackson Laboratory and bred at the NCI's Frederick Cancer Research and Development Center. Irg1 ${ }^{-/}$mice were obtained from Michael Diamond (Washington University School of Medicine, St. Louis, Missouri, USA). Mice were used at 8 to 10 weeks of age, and female mice were used for ovarian carcinoma studies.

Isolation of peritoneal $M \phi$. Peritoneal exudate cells were isolated by injecting $5 \mathrm{ml}$ sterile PBS into the peritoneal cavity, gently massaging the anesthetized mouse, and slowly withdrawing the fluid. M $\phi$ contained within the exudate fluid were purified using biotinylated anti-F4/80 antibody (clone BM8; BioLegend) and magnetic bead separation (Miltenyi Biotec). Cells were counted using a Sysmex XP-300 (Roche Diagnostics).

Cell lines. B16 melanoma, 3LL, and MC38 cell lines were obtained from the American Type Culture Collection (ATCC). The murine ovarian surface epithelial cell lines MOSEC-ID8, IG10, and IF5 were obtained from Katherine Roby (University of Kansas Medical Center, Kansas City, Kansas, USA) and grown as described previously (49). All cell lines were confirmed to be free of mycoplasma contamination. Cells were propagated in DMEM supplemented with 10\% FBS and L-glutamine. An in vivo-passaged ID8 cell line that could be reintroduced into naive mice was generated by injecting mice with $1 \times 10^{5}$ ID 8 cells and then collecting and centrifuging the resulting ascites fluid on approximately day 80 (50). For tumor cell-M $\phi$ coculture experiments, $5 \times 10^{5}$ peritoneal $\mathrm{M} \phi$ and tumor cells were mixed (1:1) and incubated in 6-well plates for 48 hours. In some experiments, tumor cells were seeded on Transwell inserts containing $3-\mu \mathrm{M}$ pores (Costar). In some experiments, tumor cells were treated with the MAPK inhibitor PD98059 (50 $\mu \mathrm{M}$; Cell Signaling Technology), and cell proliferation 
was quantified using the CellTiter Aqueous Non-Radioactive Cell Proliferation Assay (Promega).

Isolation of human samples. Peripheral blood leukocytes were isolated from healthy individuals and patients with ovarian carcinoma using Lymphocyte Separation Medium (Lonza). The ascites samples were centrifuged at 3,000 $g$ for 10 minutes to remove the noncellular ascites fluid. Red blood cells were lysed by incubating the cell pellet in ammonium chloride potassium (ACK) lysing buffer for 10 minutes. Cells were washed with PBS and cryopreserved until use. Upon thawing, the cells were counted using a Sysmex XP-300 and analyzed by flow cytometry. The number of monocytes in each bulk cell fraction was determined by multiplying the total number of cells by the percentage of CD $45^{+} \mathrm{CD} 14^{+}$cells (CD45 antibody clone HI30, CD14 antibody clone M5E2; BioLegend). In some experiments, monocytes were isolated using antibodies against CD14 and magnetically coupled beads (Miltenyi Biotec). Total RNA and cDNA used for qPCR analyses were prepared as described below.

Lentiviral shRNA generation. The In-fusion Cloning Kit (Clontech Laboratories) was used to modify the previously described $\mathrm{pHR}^{\prime}$ SIN-cPPT-SEW plasmid (51). Lentiviral particles were produced by transfecting 293-T cells with lentiviral plasmid and helper constructs (52) (pCMV- $\Delta 8.91$ [GAG/POL, Tat and Rev] and pMD2.G [VSV-G coat]) using X-tremeGENE 9 Transfection Reagent (Sigma-Aldrich). Lentivirus was concentrated using the Lenti-X Concentrator Kit (Clontech) with supernatants from 293T cells 48 hours after transfection. Lentiviral particles were resuspended in PBS and stored at $-80^{\circ} \mathrm{C}$. Mice were injected i.p. 7 days prior to tumor cell injections (day -7 ) with $200 \mu$ l concentrated lentiviral particles.

In vivo tumor model. Mice were injected i.p. with $1 \times 10^{5}$ tumor cells in $100 \mu \mathrm{l}$ sterile saline. Some mice received $100 \mu \mathrm{l}$ control or clodronate liposomes i.p. (clodronateliposomes.org) or 150 $\mathrm{mg} / \mathrm{kg}$ NAC (Sigma-Aldrich) via the drinking water. Peritoneal lavages were done on day 9 unless otherwise indicated. For the adoptive transfer experiment, peritoneal lavage exudate cells from $10 \mathrm{WT}$ or $\operatorname{Irg} 1^{-/}$mice were injected i.p. into clodronatedepleted, CD45 congenic mice 1 day prior to tumor inoculation. For quantitation of B16 melanoma cells, the tumors throughout the peritoneal cavity were surgically dissected, passed through Filtra-Bags (Labplas) and 100- $\mu \mathrm{M}$ nylon filters (Thermo Fisher Scientific), and the resulting single-cell suspension was counted on a Cellometer Auto T4 (Nexcelom Bioscience). For the in vivo-passaged ID8 tumor model, tumors were weighed and measured using calipers. Extracellular ROS contained within peritoneal lavage fluid was measured using the Amplex Red Hydrogen Peroxide/ Peroxidase Assay Kit (Thermo Fisher Scientific).

In vivo MRI. The MRI experiments were conducted using a 3.0T Intera Achieva Clinical Scanner (Philips) equipped with a 40-mmdiameter solenoid receiver coil (Philips Research) to detect the initial point of the peritoneal tumor growth and monitor its progression over time.

A multislice, T2-weighted turbospin echo (T2w-TSE) imaging sequence was applied in the coronal direction with a field of view (FOV) of $50 \times 30 \times 12 \mathrm{~mm}$ to cover the mid-part of the abdominal area. To minimize motion-induced artifacts in the resulting image, a respiratory triggering technique was used. The images were obtained with a repetition time (TR) of $5333 \mathrm{~ms}$, an echo time (TE) of $80 \mathrm{~ms}$, an in-plane resolution of $0.180 \times 0.180 \mathrm{~mm}$, and a slice thickness of 0.5 $\mathrm{mm}$. A fat suppression technique, spectral presaturation with inversion recovery (SPIR), was used to suppress the fat component in the images and thus to help distinguish fat from tumor tissue in the peritoneal cavity. The MRI methodology described generates high-resolution T2w images with a clear contrast between the tumor mass and the peritoneal organs and allows post-imaging analysis for a quantitative assessment of tumor burden.

Primary metabolite analysis. Magnetically sorted peritoneal $\mathrm{M} \phi$ $\left(5.0 \times 10^{6}\right.$ to $\left.7.5 \times 10^{6}\right)$ were pelleted and snap-frozen in liquid nitrogen. Samples were further processed and analyzed for untargeted primary metabolites by the West Coast Metabolomic Center (UCD, Davis, California, USA). Mass spectrometric gas chromatography-time of flight (GC-TOF) analysis was performed using the Agilent GC 6890/LECO Pegasus III instrument. Samples were normalized using the sum of the peak heights for all identified metabolites. Metabolite data were analyzed using IPA.

Extracellular flux analysis. Magnetically sorted resident $\mathrm{M} \phi$ were seeded at $1.0 \times 10^{6}$ (24-well) or $0.3 \times 10^{6}$ (96-well) cells per well in complete media $(+0.5 \mu \mathrm{M}$ retinoic acid, $+20 \mathrm{ng} / \mathrm{ml} \mathrm{M-CSF})$ and incubated for 2 hours. Plated cells were washed gently with PBS and incubated with Seahorse Bioscience assay media supplemented with $2 \mathrm{mM}$ glutamine and $25 \mathrm{mM}$ glucose $(+0.5 \mu \mathrm{M}$ retinoic acid, $+20 \mathrm{ng} / \mathrm{ml} \mathrm{M-CSF}$ ) for 1 hour at $37^{\circ} \mathrm{C}$ with no $\mathrm{CO}_{2}$. Extracellular flux analysis was performed at $37^{\circ} \mathrm{C}$ with no $\mathrm{CO}_{2}$ using the XF-24 or XF-96 analyzer (Seahorse Bioscience) according to the manufacturer's instructions. Port additions and times were used as indicated in the figures.

Flow cytometric analysis. Cells $\left(1 \times 10^{6}\right)$ were incubated for 15 minutes in cell-staining buffer ( $0.1 \%$ BSA, $0.1 \%$ sodium azide) containing $250 \mu \mathrm{g} / \mathrm{ml} 2.4 \mathrm{G} 2$ ascites. In some experiments, cells were incubated with $5 \mu \mathrm{M}$ CM-H2DCFDA or MitoSOX Red mitochondrial Superoxide Indicator (Invitrogen, Thermo Fisher Scientific) for 20 minutes at $37^{\circ} \mathrm{C}$. Cells were stained with fluorescently conjugated antibodies (F4/80 antibody clone BM8; CD49f antibody clone GoH3; Ly6C antibody clone AL-21; BD Pharmingen) for 20 minutes. For intracellular staining, surface-labeled cells were fixed and permeabilized (Invitrogen, Thermo Fisher Scientific) and incubated with Gata6 antibody (clone D61E4; Cell Signaling Technology) or p-ERK1/2 (Thr202/Tyr204) antibody (clone 4B11B69; BioLegend). After washing, the labeled cells were analyzed on an LSR II Flow Cytometer using FACSDIVA software (BD Biosciences).

$q P C R$. Total RNA was isolated using a High Pure RNA Isolation Kit (Roche Diagnostics). RNA was reverse transcribed using the High Capacity cDNA Archive Kit (Applied Biosystems). Genes of interest were examined by gene expression assays (Applied Biosystems). Briefly, 10 ng cDNA was placed into a final volume of $20 \mu$ l containing $10 \mu \mathrm{l}$ TaqMan Universal PCR Mix (Applied Biosystems, Thermo Fisher Scientific) and $1 \mu \mathrm{l}$ primer/probe gene expression assay. All samples were run on an ABI 7300 Real-Time PCR system and analyzed using the $\Delta \Delta \mathrm{Ct}$ method (53). Gene expression was normalized to levels of the housekeeping gene HPRT.

Western blot analysis. Purified monocytes and $\mathrm{M} \phi$ were lysed in RIPA buffer. Tumors were homogenized in RIPA buffer using a GentleMACS Tissue Dissociator (Miltenyi Biotec). Total protein was quantitated by bicinchoninic acid assay (Thermo Fisher Scientific). Total protein (10-20 $\mu \mathrm{g} /$ lane) was separated by SDS-PAGE and transferred to PVDF membranes (Invitrogen, Thermo Fisher Scientific). 
Membranes were blocked for 1 hour with 5\% BSA in TBS plus $0.05 \%$ Tween 20 and incubated overnight at $4^{\circ} \mathrm{C}$ with a primary antibody against IRG1 (Abcam), total or p-p44 or p-p42 MAPK (ERK1/2) (Cell Signaling Technology). Washed membranes were incubated with HRP-conjugated anti-mouse IgG secondary antibody and visualized using ECL (Invitrogen, Thermo Fisher Scientific).

Statistics. Statistical differences between groups were analyzed using GraphPad Prism software. A $P$ value of less than 0.05 was considered statistically significant. The tests used include ANOVA with Tukey's multiple comparisons test, Mann Whitney $U$ test, Student's $t$ test.

Study approval. All mice were used in accordance with an approved NCI Frederick IACUC protocol. All patients provided written, informed consent at study enrollment. Peripheral blood was collected from 6 healthy individuals and 5 patients with ovarian carcinoma. Peritoneal ascites fluid was collected from 11 patients with ovarian carcinoma, who had fluid removed for therapeutic purposes, and deidentified. The NIH Office of Human Subjects Research determined that federal regulations for the protection of human subjects did not apply to this study, based on the Code of Federal Regulations, Title 45, Part 46.

\section{Author contributions}

JMW designed and performed experiments and wrote the manuscript; LCD contributed to the interpretation of the results; MK performed experiments; LI performed experiments; MKO provided patients' samples; RYSC provided reagents; LAR contributed to the interpretation of the results; CMA provided patients' samples; DAW contributed to the interpretation of the results; DWM contributed to the interpretation of the results and wrote the manuscript.

\section{Acknowledgments}

$\mathrm{Irg1}^{-/-}$mice were provided by Michael Diamond (Washington University School of Medicine). Lentiviral shRNA constructs were sequenced by the CCR Genomics Core at the NCI, NIH. This research was supported by the Intramural Research Program of the NIH, NCI, CCR. LCD is funded by the Henry Wellcome Trust, United Kingdom (WT103973MA).

Address correspondence to: Daniel W. McVicar, NCI Frederick, Building 560, Room 21-89B, Frederick, Maryland, 21702-1201, USA. Phone: 301.846.5163; Email: mcvicard@mail.nih.gov.
1. Romero I, Bast RC. Minireview: human ovarian cancer: biology, current management, and paths to personalizing therapy. Endocrinology. 2012;153(4):1593-1602.

2. Davies LC, Jenkins SJ, Allen JE, Taylor PR. Tissue-resident macrophages. Nat Immunol. 2013;14(10):986-995.

3. Davies LC, Rice CM, Palmieri EM, Taylor PR, Kuhns DB, McVicar DW. Peritoneal tissueresident macrophages are metabolically poised to engage microbes using tissue-niche fuels. Nat Commun. 2017;8(1):2074.

4. Ruffell B, Affara NI, Coussens LM. Differential macrophage programming in the tumor microenvironment. Trends Immunol. 2012;33(3):119-126.

5. Robinson-Smith TM, et al. Macrophages mediate inflammation-enhanced metastasis of ovarian tumors in mice. Cancer Res. 2007;67(12):5708-5716.

6. Moughon DL, et al. Macrophage Blockade Using CSF1R Inhibitors Reverses the Vascular Leakage Underlying Malignant Ascites in LateStage Epithelial Ovarian Cancer. Cancer Res. 2015;75(22):4742-4752.

7. Chang $\mathrm{CH}$, et al. Metabolic competition in the tumor microenvironment is a driver of cancer progression. Cell. 2015;162(6):1229-1241.

8. Colegio OR, et al. Functional polarization of tumour-associated macrophages by tumourderived lactic acid. Nature. 2014;513(7519):559-563.

9. Biswas SK. Metabolic reprogramming of immune cells in cancer progression. Immunity. 2015;43(3):435-449.

10. Biswas SK, Mantovani A. Orchestration of metabolism by macrophages. Cell Metab. 2012;15(4):432-437.

11. Izquierdo E, et al. Reshaping of Human Macrophage Polarization through Modulation of Glucose Catabolic Pathways. J Immunol. 2015;195(5):2442-2451.

12. Kelly B, O'Neill LA. Metabolic reprogramming in macrophages and dendritic cells in innate immunity. Cell Res. 2015;25(7):771-784.

13. Meiser J, et al. Pro-inflammatory Macrophages Sustain Pyruvate Oxidation through Pyruvate Dehydrogenase for the Synthesis of Itaconate and to Enable Cytokine Expression. J Biol Chem. 2016;291(8):3932-3946.

14. Mills EL, et al. Succinate Dehydrogenase Supports Metabolic Repurposing of Mitochondria to Drive Inflammatory Macrophages. Cell. 2016;167(2):457-470.e13.

15. Degrandi D, Hoffmann R, Beuter-Gunia C, Pfeffer K. The proinflammatory cytokine-induced IRG1 protein associates with mitochondria. JInterferon Cytokine Res. 2009;29(1):55-67.

16. Strelko CL, et al. Itaconic acid is a mammalian metabolite induced during macrophage activation. JAm Chem Soc. 2011;133(41):16386-16389.

17. Cordes T, Michelucci A, Hiller K. Itaconic Acid: The Surprising Role of an Industrial Compound as a Mammalian Antimicrobial Metabolite. Annu Rev Nutr. 2015;35:451-473.

18. Cordes T, et al. Immunoresponsive Gene 1 and Itaconate Inhibit Succinate Dehydrogenase to Modulate Intracellular Succinate Levels. J Biol Chem. 2016;291(27):14274-14284.

19. Lampropoulou V, et al. Itaconate Links Inhibition of Succinate Dehydrogenase with Macrophage Metabolic Remodeling and Regulation of Inflammation. Cell Metab. 2016;24(1):158-166.

20. Michelucci A, et al. Immune-responsive gene 1 protein links metabolism to immunity by catalyzing itaconic acid production. Proc Natl Acad Sci US A. 2013;110(19):7820-7825

21. Tallam A, et al. Gene regulatory network inference of immunoresponsive gene 1 (IRG1) identifies interferon regulatory factor 1 (IRF1) as its transcriptional regulator in mammalian macrophages. PLoS One. 2016;11(2):e0149050.

22. Chen B, Zhang D, Pollard JW. Progesterone regulation of the mammalian ortholog of methylcitrate dehydratase (immune response gene 1) in the uterine epithelium during implantation through the protein kinase $\mathrm{C}$ pathway. $\mathrm{Mol}$ Endocrinol. 2003;17(11):2340-2354.

23. Hall CJ, et al. Immunoresponsive gene 1 augments bactericidal activity of macrophage-lineage cells by regulating $\beta$-oxidation-dependent mitochondrial ROS production. Cell Metab. 2013;18(2):265-278.

24. Ren K, et al. Suppression of IRG-1 Reduces Inflammatory Cell Infiltration and Lung Injury in Respiratory Syncytial Virus Infection by Reducing Production of Reactive Oxygen Species. J Virol. 2016;90(16):7313-7322.

25. Gupta SC, Hevia D, Patchva S, Park B, Koh W, Aggarwal BB. Upsides and downsides of reactive oxygen species for cancer: the roles of reactive oxygen species in tumorigenesis, prevention, and therapy. Antioxid Redox Signal. 2012;16(11):1295-1322.

26. Liou GY, Storz P. Reactive oxygen species in cancer. Free Radic Res. 2010;44(5):479-496.

27. Wittgen HG, van Kempen LC. Reactive oxygen species in melanoma and its therapeutic implications. Melanoma Res. 2007;17(6):400-409.

28. Brar SS, et al. Reactive oxygen species from NAD(P) $\mathrm{H}$ :quinone oxidoreductase constitutively activate NF-kappaB in malignant melanoma cells. Am J Physiol, Cell Physiol. 2001;280(3):C659-C676.

29. Lin X, et al. Oxidative stress in malignant melanoma enhances tumor necrosis factor- $\alpha$ secretion of tumor-associated macrophages that promote cancer cell invasion. Antioxid Redox Signal. 2013;19(12):1337-1355.

30. Cassado Ados A, D’Império Lima MR, Bortoluci KR. Revisiting mouse peritoneal macrophages: heterogeneity, development, and function. Front Immunol. 2015;6:225.

31. Gautier EL, et al. Gata 6 regulates aspartoacylase expression in resident peritoneal macrophages and controls their survival. J Exp Med. 2014;211(8):1525-1531.

32. Rosas M, et al. The transcription factor Gata6 
links tissue macrophage phenotype and proliferative renewal. Science. 2014;344(6184):645-648.

33. Murray PJ, et al. Macrophage activation and polarization: nomenclature and experimental guidelines. Immunity. 2014;41(1):14-20.

34. Huang SC, et al. Cell-intrinsic lysosomal lipolysis is essential for alternative activation of macrophages. Nat Immunol. 2014;15(9):846-855.

35. Soda K. The mechanisms by which polyamines accelerate tumor spread. J Exp Clin Cancer Res. 2011;30:95.

36. Kalyanaraman B, et al. Measuring reactive oxygen and nitrogen species with fluorescent probes: challenges and limitations. Free Radic Biol Med. 2012;52(1):1-6.

37. Hoos MD, et al. The impact of human and mouse differences in NOS2 gene expression on the brain's redox and immune environment. $\mathrm{Mol}$ Neurodegener. 2014;9:50.

38. Cheon YP, Xu X, Bagchi MK, Bagchi IC. Immune-responsive gene 1 is a novel target of progesterone receptor and plays a critical role during implantation in the mouse. Endocrinology. 2003;144(12):5623-5630.

39. Li Y, et al. Immune responsive gene 1 (IRG1) promotes endotoxin tolerance by increasing A20 expression in macrophages through reactive oxygen species. J Biol Chem. 2013;288(23):16225-16234.

40. Pan J, et al. Immune responsive gene 1 , a novel oncogene, increases the growth and tumorigenicity of glioma. Oncol Rep. 2014;32(5):1957-1966.

41. Jamal Uddin M, et al. IRG1 induced by heme oxygenase-1/carbon monoxide inhibits LPS-mediated sepsis and pro-inflammatory cytokine production. Cell Mol Immunol. 2016;13(2):170-179.

42. Jozkowicz A, Was H, Dulak J. Heme oxygenase-1 in tumors: is it a false friend? Antioxid Redox Signal. 2007;9(12):2099-2117.

43. Palmieri F. The mitochondrial transporter family SLC25: identification, properties and physiopathology. Mol Aspects Med. 2013;34(2-3):465-484.

44. Murphy MP. How mitochondria produce reactive oxygen species. Biochem J. 2009;417(1):1-13.

45. Hossain F, et al. Inhibition of fatty acid oxidation modulates immunosuppressive functions of myeloid-derived suppressor cells and enhances cancer therapies. Cancer Immunol Res. 2015;3(11):1236-1247.

46. Sasikaran J, Ziemski M, Zadora PK, Fleig A, Berg IA. Bacterial itaconate degradation promotes pathogenicity. Nat Chem Biol. 2014;10(5):371-377.

47. Wang SF, Adler J, Lardy HA. The pathway of itaconate metabolism by liver mitochondria. J Biol Chem.
1961;236:26-30.

48. Sakai A, Kusumoto A, Kiso Y, Furuya E. Itaconate reduces visceral fat by inhibiting fructose 2,6-bisphosphate synthesis in rat liver. Nutrition. 2004;20(11-12):997-1002.

49. Roby KF, et al. Development of a syngeneic mouse model for events related to ovarian cancer. Carcinogenesis. 2000;21(4):585-591.

50. Janát-Amsbury MM, Yockman JW, Anderson ML, Kieback DG, Kim SW. Comparison of ID8 MOSE and VEGF-modified ID8 cell lines in an immunocompetent animal model for human ovarian cancer. Anticancer Res. 2006;26(4B):2785-2789.

51. Demaison C, et al. High-level transduction and gene expression in hematopoietic repopulating cells using a human immunodeficiency [correction of imunodeficiency] virus type 1-based lentiviral vector containing an internal spleen focus forming virus promoter. Hum Gene Ther. 2002;13(7):803-813.

52. Zufferey R, Nagy D, Mandel RJ, Naldini L, Trono D. Multiply attenuated lentiviral vector achieves efficient gene delivery in vivo. Nat Biotechnol. 1997;15(9):871-875.

53. Livak KJ, Schmittgen TD. Analysis of relative gene expression data using real-time quantitative PCR and the 2(-Delta Delta C(T)) Method. Methods. 2001;25(4):402-408. 